\title{
Gemella morbillorum endocarditis in a patient with a bicuspid aortic valve
}

\author{
(D) Mustafa Dogan, ${ }^{1}$ (D) Aynur Eren Topkaya, ${ }^{2}$ (D) Seref Alpsoy, ${ }^{3}$ (D) Ozcan Gur, ${ }^{4}$ (D) Ilknur Erdem ${ }^{1}$ \\ ${ }^{1}$ Department of Infectious Diseases, Namik Kemal University Faculty of Medicine, Tekirdag, Turkey \\ ${ }^{2}$ Department of Medical Microbiology, Namik Kemal University Faculty of Medicine Tekirdag, Turkey \\ ${ }^{3}$ Department of Cardiology, Namik Kemal University Faculty of Medicine Tekirdag, Turkey \\ ${ }^{4}$ Department of Cardiovascular Surgery, Namik Kemal University Faculty of Medicine Tekirdag, Turkey
}

\begin{abstract}
Gemella morbillorum is one of the rare causative microorganisms of endocarditis. We herein report a case of infective endocarditis in a patient with bicuspid aortic valve caused by G. morbillorum. Infective endocarditis diagnosis was established based on the Modified Duke's criteria. The patient was successfully treated with medical-surgical management.
\end{abstract}

Keywords: Bicuspid aortic valve; endocarditis; Gemella morbillorum.

Cite this article as: Dogan M, Eren Topkaya A, Alpsoy S, Gur O, Erdem I. Gemella morbillorum endocarditis in a patient with a bicuspid aortic valve. North Clin Istanb 2021;8(2):190-192.

\begin{abstract}
Cemella morbillorum endocarditis is rare condition, with few cases reported in the English literature. Despite the improvements in the treatment methods, the morbidity and mortality rates are still high. Delays of weeks or months in antimicrobial treatment increase the risk of the occurrence embolic or immunological events $[1,2]$. In this paper, it was reported a case of native bicuspid aortic and mitral valve endocarditis caused by $G$. morbillorum.
\end{abstract}

\section{CASE REPORT}

A 37-year-old male patient with the complains of fever, fatigue, sweating, and loss of more than $10 \%$ of his body weight for 1 month. Right upper quadrant pain and high fever was began 1 week ago. The patient had no history of smoking, alcohol consumption, or illicit drug use. No history of heart valve disease was ascertained. He was a workman in a chemical factory. On his physical exam- ination, body temperature was $38.6^{\circ} \mathrm{C}$, pulse rate was 112 beats/min, respiratory rate was 38 breaths/min, and blood pressure was $90 / 70 \mathrm{mmHg}$. Cardiac examination revealed a 3/6 pansystolic murmur. The liver was palpable $2 \mathrm{~cm}$ below the right costal margin and his traube's space was closed. In addition to the available findings, splinter hemorrhage was seen on the fourth finger of the left hand. Laboratory findings were as follows: White blood count, $4300 / \mathrm{mm}^{3}$ (68\% PNL); hemoglobin, 7.9 $\mathrm{g} / \mathrm{d}$; platelets, $81,000 / \mathrm{mm}^{3}$; C-reactive protein, 102 $\mathrm{mg} / \mathrm{L}$ (RR 0-5 mg/L); erythrocyte sedimentation rate, 122/h; and albumin, $2.8 \mathrm{~g} / \mathrm{dL}$ (RR 3.5-5.5 g/dL). Liver and kidney function tests were normal. Three sets of blood culture and bone marrow aspirate were obtained from the patient who was hospitalized to investigate the cause of fever. Intravenous ceftriaxone $(1 \times 2 \mathrm{~g} /$ day $)$ was started empirically after blood cultures are taken.

Transthoracic echocardiography revealed vegetation on bicuspid aortic valve which was a $22 \times 18 \mathrm{~mm}$ in di-

Received: December 11, 2019 Accepted: January 21, 2020 Online: July 23, 2020

Correspondence: Ilknur ERDEM, MD. Namik Kemal Universitesi Tip Fakultesi, Enfeksiyon Hastaliklari Anabilim Dali, Tekirdag, Turkey. Tel: +90 5325888235 e-mail: ilknurerdem@hotmail.com

(c) Copyright 2021 by Istanbul Provincial Directorate of Health - Available online at www.northclinist.com 
ameter near the left coronary cuspis. There was another vegetation as mobile mass with the diameter of $20 \times 15$ $\mathrm{mm}$ on the anterior leaflet of the mitral valve and was causing mitral insufficiency (Fig. 1). Ejection fraction was $50 \%$. Similar findings were also demonstrated in transesophageal echocardiography (TEE). In abdominal ultrasonography, the liver size was $165 \mathrm{~cm}$ and spleen size was $162 \mathrm{~cm}$.

Gram-positive cocci were yielded from three sets of blood culture bottles and bone marrow aspirate (BacT/ ALERT, bioMérieux, Marcy l'Etoile, France) after $24 \mathrm{~h}$. G. morbillorum was identified by matrix-assisted laser desorption ionization-time of flight mass spectrometry (MALDI-TOF MS, Bruker Daltonics Inc.). Antimicrobial susceptibility was performed using Kirby-Bauer disk diffusion method and an automated system according to standard guidelines. The isolate was susceptible to ampicillin, ceftriaxone, and vancomycin but was resistant to gentamicin. Infective endocarditis was diagnosed based on the modified Duke criteria of one major criterion (evidence of endocardial involvement) and four minor criteria (positive blood cultures, fever, bicuspid aortic valve, and evidence of septic emboli).

On the $15^{\text {th }}$ day of the treatment, control TEE showed no regression of vegetation size. The patient was consulted by the cardiovascular surgery clinic. Mitral and aortic valve replacement was performed. The patient was discharged after 28 days of antibiotic treatment.

\section{DISCUSSION}

G. morbillorum (formerly Streptococcus morbillorum), which was first described in 1917 by Tunnicliffe, was classified as a separate bacterial strain with its biomolecular and physiological properties in 1988 [2]. G. morbillorum possess Gram-positive cell wall structure, but it also has a feature of Gram-variable probably due to their relatively thin cell walls $[3,4]$. It is part of the normal flora of the oral cavity, gastrointestinal, and genitourinary tract of the human body $[4,5]$.

G. morbillorum may cause dental abscess, pleural empyema, liver abscess, peritonitis, pericarditis, mediastinitis, sepsis, spondylodiscitis, soft tissue infection, meningitis, brain abscesses, and infective endocarditis as an opportunistic agent. It is not a common infectious agent and usually requires the presence of various predisposing factors to become as infectious pathogen. These predisposing factors include poor dental hy-

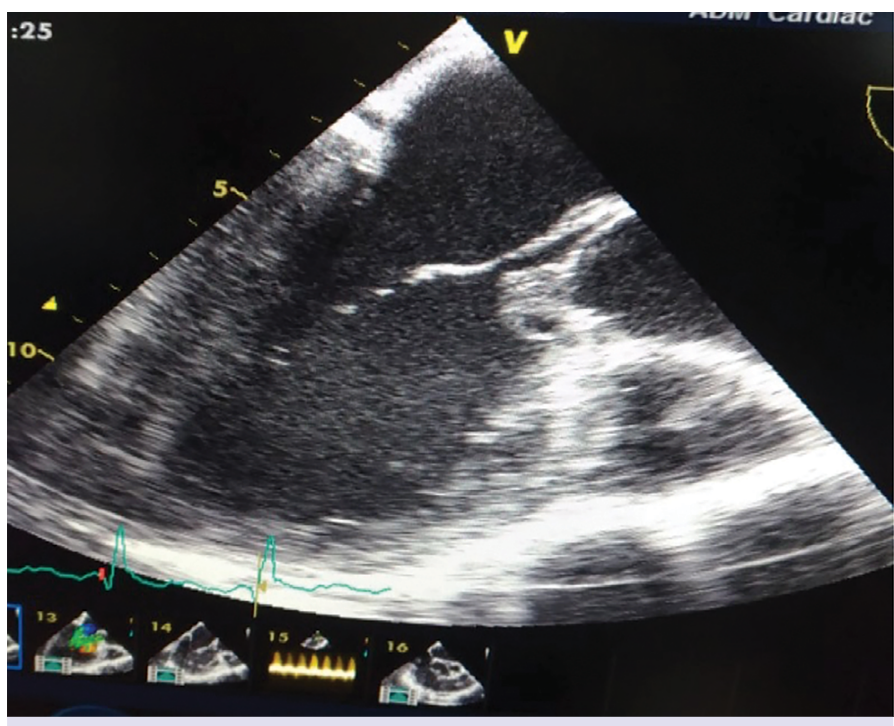

FIGURE 1. Transthoracic echocardiography showing the vegetation on the bicuspid aortic valve and the mitral valve.

giene, dental procedures, gastrointestinal procedures, colon malignancies, and underlying cardiac pathologies such as rheumatic heart disease, surgically repaired intracardiac lesions, and congenitally bicuspid aortic valve [4-7].

It is reported in a limited number of cases as endocarditis, although endocarditis is the most common clinical manifestation of $G$. morbillorum. Both native and prosthetic valves can be affected. Aortic valve was more frequently affected than mitral valve [7-9]. Bicuspid aortic valve is the most common congenital heart anomaly with an estimated incidence of up to $0.9-2 \%$ in the general population $[9,10]$. In our patient, it is mentioned that bicuspid aorta is an important predisposing factor. There were present both aortic valve vegetation and $\mathrm{mi}$ tral valve vegetation.

In the medical treatment of infective endocarditis due to $G$. morbillorum, different options were applied taking into account the sensitivity of the agent. Antibiotics were administered between 4 and 6 weeks in alone penicillin $\mathrm{G}$, ceftriaxone, vancomycin, or combination with gentamicin and rifampin $[4,5]$. In the treatment of our case was started once a day with intravenous, $2 \mathrm{~g}$ ceftriaxone. According to the antibiogram results, ceftriaxone was continued without any change in treatment.

G. morbillorum can cause rapid destruction in the heart valves and may lead to a significant clinical deterioration. In some cases, the medical treatment may not be 
shown the desired response due to the increase in regurgitation and failure of the mitral valve and aortic valve, the size of the vegetation $(>1 \mathrm{~cm} \mathrm{dm})$, and the presence of septic emboli or the bacterium's own virulence characteristics $[2,6,7,11]$. In our case, on the 15 th day of antibiotic therapy, ruptured mitral chordae tendineae, marked reduction in ejection fraction and vegetation $>1.5 \mathrm{~cm}$ in both valves were detected in TEE. Mitral valve and aortic valve replacement was performed. Antibiotic therapy was continued for 4 weeks and was seen to have improved in his clinical findings.

\section{Conclusion}

Infective endocarditis is a serious and life-threating disease. Rare pathogens such as G. morbillorum may be the cause of infective endocarditis and usually affects the immunosuppressive population; this organism can even lead to the occurrence of quite large vegetation.

Informed Consent: Written informed consent was obtained from the patient for the publication of the case report and the accompanying images.

Conflict of Interest: No conflict of interest was declared by the authors.

Financial Disclosure: The authors declared that this study has received no financial support.

Authorship Contributions: Concept - MD, IE; Design - MD, IE; Supervision - MD, AET; Fundings - MD; Materials - MD, AET, SA, OG; Data collection and/or processing - MD, AET, SA, OG, IE; Analysis and/or interpretation - MD, AET, IE; Literature review - MD, IE; Writing - MD, IE; Critical review - IE.

\section{REFERENCES}

1. Debast SB, Koot R, Meis JF. Infections caused by Gemella morbillorum. Lancet 1993;342:560. [CrossRef]

2. Ural S, Gul Yurtsever S, Ormen B, Turker N, Kaptan F, El S, Akyildiz ZI, et al. Gemella morbillorum Endocarditis. Case Rep Infect Dis 2014;2014:456471. [CrossRef]

3. Kilpper-Balz R, Schleifer KH. Transfer of Streptococcus morbillorum to the genus Gemella as Gemella morbillorum comb. nov. Int J Syst Bacteriol 1988;38:442-3. [CrossRef]

4. Kofteridis DP, Anastasopoulos T, Panagiotakis S, Kontopodis E, Samonis G. Endocarditis caused by Gemella morbillorum resistant to beta-lactams and aminoglycosides. Scand J Infect Dis 2006;38:1125-7.

5. Lopez-Dupla M, Creus C, Navarro O, Raga X. Association of Gemella morbillorum endocarditis with adenomatous polyps and carcinoma of the colon: case report and review. Clin Infect Dis 1996;22:379-80.

6. Akiyama K, Taniyasu N, Hirota J, Iba Y, Maisawa K. Recurrent aortic valve endocarditis caused by Gemella morbillorum-report of a case and review of the literature. Jpn Circ J 2001;65:997-1000. [CrossRef]

7. Warnes CA, Williams RG, Bashore TM, Child JS, Connolly HM, Dearani JA, et al. ACC/AHA 2008 Guidelines for the Management of Adults with Congenital Heart Disease: a report of the American College of Cardiology/American Heart Association Task Force on Practice Guidelines (writing committee to develop guidelines on the management of adults with congenital heart disease). Circulation 2008;118:e714-833. [CrossRef]

8. Zakir RM, Al-Dehneh A, Dabu L, Kapila R, Saric M. Mitral bioprosthetic valve endocarditis caused by an unusual microorganism, Gemella morbillorum, in an intravenous drug user. J Clin Microbiol 2004;42:4893-6. [CrossRef]

9. Ward C. Clinical significance of the bicuspid aortic valve. Heart 2000;83:81-5. [CrossRef]

10. Li JS, Sexton DJ, Mick N, Nettles R, Fowler VG Jr, Ryan T, et al. Proposed modifications to the Duke criteria for the diagnosis of infective endocarditis. Clin Infect Dis 2000;30:633-8. [CrossRef]

11. Zheng M, Ng OT, Teo BW. Aortic and mitral valve endocarditis caused by Gemella morbillorum in a haemodialysis patient. Singapore Med J 2008;49:e385-7. 